\title{
WEAK TOPOLOGIES ON SUBSPACES OF $C(S)$
}

BY

JOEL H. SHAPIRO

\begin{abstract}
Let $S$ be a locally compact Hausdorff space, $E$ a linear subspace of $C(S)$. It is shown that the unit ball of $E$ is compact in the strict topology if and only if both of the following two conditions are satisfied: (1) $E$ is the Banach space dual of $M(S) / E^{0}$ in the integration pairing, and (2) the bounded weak star topology on $E$ coincides with the strict topology. This result is applied to several examples, among which are $l^{\infty}$ and the space of bounded analytic functions on a plane region.
\end{abstract}

The space $C(S)$ of bounded, continuous, complex valued functions on the locally compact Hausdorff space $S$ is paired by integration with $M(S)$, the space of regular Borel measures on $S$ having finite variation. We call a vector subspace of $C(S)$ weakly normal if its unit ball is compact in the weak topology of this pairing, and normal if its unit ball is compact in the strict topology (see $\$ 1$ for definitions).

In $\S 1$ of this paper we show that every normal subspace of $C(S)$ is weakly normal, every weakly normal subspace is norm closed; and that a subspace is normal if and only if its unit ball is compact in the compact-open topology. We discuss several examples of normal subspaces, in particular the spaces $H^{\infty}(G)$ of bounded analytic functions on the plane region $G$, and $l^{\infty}(\Gamma)$ of bounded complex valued functions on the index set $\Gamma$.

If $E$ is a linear subspace of $C(S)$, and $E^{0}$ is its annihilator in $M(S)$, then it is easy to see that the mapping which takes $f$ in $E$ to the linear functional

$$
m+E^{0} \rightarrow \int f d m \quad(m \text { in } M(S))
$$

is a linear isometry of $E$ into the dual of $M(S) / E^{0}$. We show that $E$ is weakly normal if and only if this map is onto.

In the second section we prove our main result (Theorem 2): A subspace $E$ of $C(S)$ is normal if and only if it is weakly normal and the bounded weak star topology induced on it by $M(S) / E^{0}$ coincides with the strict topology.

In the last section we show that if $S$ is separable and $E$ is a normal subspace of $C(S)$, then a subset of $E$ is strictly closed if and only if it is strictly sequentially closed. This result was first proved by Paul Hessler for $H^{\infty}(G)$.

Our work continues that of Rubel and Shields [10] and Rubel and Ryff [9], who studied the particular class of normal subspaces $H^{\infty}(G)$. The coincidence of the

Received by the editors January 8, 1970 and, in revised form, June 29, 1970.

AMS 1969 subject classifications. Primary 4625; Secondary 4601, 4630.

Key words and phrases. Bounded continuous functions, bounded weak star topology, strict topology, equicontinuous set, bounded analytic functions, Lipschitz spaces. 
strict and bounded weak star topologies on $H^{\infty}(G)$ is due to Rubel and Ryff $[9, \S 2]$; indeed this paper arises from our attempt to better understand their result. We also make contact with the work of Collins [2] on $l^{\infty}(\Gamma)$ in the strict topology.

We wish to thank the referee for a number of insightful comments which improved the exposition and shortened the proofs of Theorem 1 and Proposition 7.

1. Normal and weakly normal subspaces. In this section we discuss some preliminary facts about normal and weakly normal subspaces, and give several examples. We begin with some definitions. If $\langle E, F\rangle$ is a dual pair of vector spaces, the weak topology induced on $E$ by $F$ is denoted by $w(E, F)$. A vector topology $t$ on $E$ is said to be consistent with the pairing $\langle E, F\rangle$ if $F$ is the $t$-dual of $E$ in the sense that a linear functional $\lambda$ on $E$ is $t$-continuous if and only if there is a unique $f_{\lambda}$ in $F$ with $\lambda(e)=\left\langle e, f_{\lambda}\right\rangle$ for each $e$ in $E$. If $E$ and $F$ are Banach spaces and the mapping $\lambda \rightarrow f_{\lambda}$ is an isometry of $E^{\prime}$, the dual of $E$, onto $F$; then we say $F$ is isometric to $E^{\prime}$ in the pairing $\langle$,$\rangle .$

Throughout our work $S$ denotes a locally compact Hausdorff space. $C(S)$ is the space of bounded, continuous, complex valued functions on $S ; C_{0}(S)$ is the subspace of $C(S)$ consisting of functions which vanish at infinity, and $M(S)$ is the space of regular complex Borel measures on $S$ of finite variation. $C(S)$ is a Banach space in the supremum norm \|\|$_{\infty}$, and $C_{0}(S)$ is a closed subspace. $M(S)$ is a Banach space in the variation norm \|\| , and is isometric to the dual of $C_{0}(S)$ in the pairing

$$
\langle f, m\rangle=\int f d m .
$$

The strict topology $\beta$ on $C(S)$ is the locally convex topology defined by the seminorms

$$
\|f\|_{k}=\|f k\|_{\infty} \quad(f \text { in } C(S)),
$$

where $k$ runs through $C_{0}(S)$. The strict topology is Hausdorff, complete, and consistent with the pairing (1.1) between $C(S)$ and $M(S)$ [1, Theorems 1 and 2]. Moreover, it coincides with the norm topology if and only if $S$ is compact [1, Theorem 1]. We write $\alpha=w(C(S), M(S))$.

The following elementary fact is used repeatedly:

THEOREM A. Suppose $t_{1}$ and $t_{2}$ are Hausdorff topologies on a set $X$. If $t_{1} \subset t_{2}$ and $\left(X, t_{2}\right)$ is compact, then $\left(X, t_{1}\right)$ is compact and $t_{1}=t_{2}$.

We call a vector subspace of $C(S)$ weakly normal if its unit ball is $\alpha$-compact, and normal if its unit ball is $\beta$-compact.

Proposition 1. Every normal subspace of $C(S)$ is weakly normal, and every weakly normal subspace is norm closed.

Proof. If $E$ is normal then its unit ball $E_{1}$ is $\beta$-compact. Since $\beta$ is consistent with the pairing $\langle C(S), M(S)\rangle, \alpha$ is weaker than $\beta$. Thus $E_{1}$ is $\alpha$-compact by Theorem A, 
so $E$ is weakly normal. If $E$ is weakly normal then $E_{1}$ is $\alpha$-compact, hence $\alpha$-closed in $C(S)$. Since $\alpha$ is weaker than the norm topology, $E_{1}$ is norm closed in $C(S)$, and it follows easily that the same is true of $E$.

A weakly normal subspace need not be normal. For example, if $S$ is the closed unit interval and $E$ is a reflexive infinite-dimensional subspace of $C(S)$, then $\alpha$ coincides on $E$ with $w\left(E, E^{\prime}\right)$, so the unit ball of $E$ is $\alpha$-compact. However, $E$ is not normal, since in this example the strict topology coincides with the norm topology.

The next result gives a useful characterization of normality. Let $\kappa$ denote the compact-open topology on $C(S)$.

Proposition 2. A vector subspace of $C(S)$ is normal if and only if its unit ball is equicontinuous and $\kappa$-closed.

Proof. Let $E$ be a vector subspace of $C(S)$, and $E_{1}$ its unit ball. In view of [1, Theorem 1], $\kappa=\beta$ on $E_{1}$, hence $E$ is normal if and only if $E_{1}$ is $\kappa$-compact. The result now follows from the Arzela-Ascoli Theorem and the fact that the unit ball of $C(S)$ is $\kappa$-closed in the space of all continuous functions on $S$.

COROLlaRY. $H^{\infty}(G)$ is a normal subspace of $C(G)$.

Let $\Gamma$ be an index set, and let $S$ denote $\Gamma$ in its discrete topology. Then $l^{\infty}(\Gamma)$ $=C(S)$ is normal, as the following result shows.

Proposition 3. The following are equivalent:

(a) $S$ is a discrete space.

(b) $C(S)$ is normal.

(c) $C(S)$ is weakly normal.

Proof. (a) $\rightarrow$ (b). It follows from (a) that $C(S)$ is the space of all bounded functions on $S$, so (b) is an easy consequence of Proposition 2.

(b) $\rightarrow$ (c). Immediate from Proposition 1 .

(c) $\rightarrow$ (a). Suppose $C(S)$ is weakly normal. Fix $s$ in $S$. Let $U$ be an open set containing $S$, and apply Urysohn's Lemma to the one point compactification of $S$ to obtain a continuous function $f_{U}$ vanishing on the complement of $U$, with $f_{U}(s)=1$ and $0 \leqq f_{U} \leqq 1$. Evidently the net $\left(f_{U}\right)$ converges pointwise to $\phi_{s}$, the characteristic function of the singleton $\{s\}$. However, $\left(f_{U}\right)$ is contained in the $\alpha$-compact unit ball of $C(S)$, so there is a function $g$ in $C(S)$ and a subnet of $\left(f_{U}\right)$ which is $\alpha$-convergent, hence pointwise convergent, to $g$. Thus $g=\phi_{s}$, so $\phi_{s}$ is continuous on $S$. It follows that $\{s\}$ is open in $S$, which completes the proof.

The next example was pointed out to us by D. L. Williams. Let $\Lambda$ be a compact subset of $n$-dimensional Euclidean space $R^{n}$, and let $E(\Lambda)$ denote the space of bounded measurable functions on $R^{n}$ with spectrum [4, p. 227] contained in $\Lambda$. Every such function is the restriction to $R^{n}$ of an entire function on $C^{n}$ of exponential type, and the Bernstein inequality guarantees that

$$
\|\operatorname{grad} f\|_{\infty} \leqq A\|f\|_{\infty} \quad(f \text { in } E(\Lambda)),
$$


for some $A>0$ independent of $f$ [4, p. 227]. It follows from the mean value theorem in $R^{n}$ that

$$
|f(y)-f(x)| \leqq\|\operatorname{grad} f\|_{\infty}|y-x| \quad\left(x, y \text { in } R^{n}\right)
$$

for $f$ in $C^{1}\left(R^{n}\right)$. Thus for $f$ in the unit ball of $E(\Lambda)$, the Bernstein inequality yields $|f(y)-f(x)| \leqq A|y-x|$, where $A$ is independent of $f, x$, and $y$. The unit ball of $E(\Lambda)$ is therefore equicontinuous. That it is $\kappa$-closed follows from a routine argument involving Fourier transforms of temperate distributions. By Proposition 2, $E(\Lambda)$ is therefore a normal subspace of $C\left(R^{n}\right)$.

Finally let $\operatorname{Lip}(X, d)$ denote the space of Lipschitz functions on the compact metric space $(X, d)$. More explicitly, $\operatorname{Lip}(X, d)$ consists of all those continuous complex-valued functions on $X$ for which

$$
\omega f(x, y)=(f(x)-f(y)) / d(x, y)
$$

is bounded for $x \neq y(x, y$ in $X)$. Let

$$
\|\omega f\|_{\infty}=\sup \{|\omega f(x, y)|: x, y \text { in } X, x \neq y\} .
$$

In the norm $\|f\|=\max \left\{\|f\|_{\infty},\|\omega f\|_{\infty}\right\}$, Lip $(X, d)$ is a Banach space. Following de Leeuw [3] and Johnson [7, Lemma 3.1] we show that it can be regarded as a normal subspace of an appropriate $C(S)$.

Let $Y=\{(x, y): x, y$ in $X, x \neq y\}$ be given the relative topology of $X \times X$. Then the disjoint union $W=X \cup Y$ in its natural topology is a locally compact Hausdorff space. For $f$ in $\operatorname{Lip}(X, d)$ define $\tilde{f}$ on $W$ by setting $\tilde{f}$ equal to $f$ on $X$, and to $\omega f$ on $Y$. The mapping $f \rightarrow \tilde{f}$ is then a linear isometry of $\operatorname{Lip}(X, d)$ onto a subspace $L$ of $C(W)$, and it is not difficult to show from the Lipschitz condition that the unit ball of $L$ is equicontinuous. It is clearly $\kappa$-closed in $C(W)$, so $\operatorname{Lip}(X, d)$ may be regarded as a normal subspace of $C(W)$.

We close this section by characterizing weakly normal subspaces as special conjugate Banach spaces. For this we need the Krein-Smulian Theorem.

Theorem B (Krein-Šmulian [5, V.5.7]). Let $X$ be a Banach space. A linear subspace of $X^{\prime}$ is $w\left(X^{\prime}, X\right)$-closed if and only if its intersection with the $X^{\prime}$ unit ball is $w\left(X^{\prime}, X\right)$-closed.

Let $E$ be a vector subspace of $C(S)$, and let $E^{0}$ be the annihilator of $E$ in $M(S)$. $E$ is paired with the quotient space $M(S) / E^{0}$ by integration:

$$
\left\langle m+E^{0}, f\right\rangle=\int f d m \quad(m \text { in } M(S), f \text { in } E),
$$

and the linear functional

$$
m+E^{0} \rightarrow\left\langle m+E^{0}, f\right\rangle
$$

on $M(S) / E^{0}$ is easily seen to have norm $\|f\|_{\infty}$. Thus the mapping which takes $f$ in $E$ to the linear functional (1.3) is a linear isometry of $E$ onto a weak star dense subspace of the dual of $M(S) / E^{0}$. 
THEOREM 1. E is weakly normal if and only if it is isometric in the pairing (1.2) to the dual of $M(S) / E^{0}$.

Proof. Let $M=M(S)$ and let $E_{1}$ denote the unit ball of $E$. If $E$ is isometric to $\left(M / E^{0}\right)^{\prime}$ in (1.2) then it follows from the Alaoglu Theorem that $E_{1}$ is $w\left(E, M / E^{0}\right)$ compact. Since $w\left(E, M / E^{0}\right)=\alpha$ on $E, E$ is weakly normal.

Conversely, suppose $E$ is weakly normal. Identifying $E$ with its image in $\left(M / E^{0}\right)^{\prime}$, we see by weak normality that the unit ball of $E$ is $\alpha=w\left(E, M / E^{0}\right)$-compact, so the Krein-Šmulian Theorem guarantees that $E$ is weak star closed in $\left(M / E^{0}\right)^{\prime}$. But we have already seen that $E$ is weak star dense in $\left(M / E^{0}\right)^{\prime}$, so the proof is complete.

2. Normal subspaces and the bounded weak star topology. If $X$ is a Banach space, then the bounded weak star topology $b w\left(X^{\prime}, X\right)$ on its dual $X^{\prime}$ is the strongest topology which coincides with $w\left(X^{\prime}, X\right)$ on bounded subsets. A subset of $X^{\prime}$ is $b w\left(X^{\prime}, X\right)$-closed if and only if its intersection with every positive multiple of the $X^{\prime}$ unit ball is $w\left(X^{\prime}, X\right)$-closed [5, V.5.3]. For example Theorem B can be restated: a linear subspace of $X^{\prime}$ is $w\left(X^{\prime}, X\right)$-closed if and only if it is $b w\left(X^{\prime}, X\right)$-closed. The bounded weak star topology has the following important characterization:

THEOREM C [5, V.5.4]. If $X$ is a Banach space then $b w\left(X^{\prime}, X\right)$ coincides with the topology of uniform convergence on sequences in $X$ which tend in norm to zero.

We turn to our main result. Recall that a subspace $E$ of $C(S)$ is weakly normal if and only if it is isometric to the dual of $M(S) / E^{0}$ in the pairing (1.2). Thus if $E$ is weakly normal, then its predual $M(S) / E^{0}$ induces on it the weak star topology (which coincides with $\alpha$ ), and the bounded weak star topology $b w\left(E, M(S) / E^{0}\right)$.

THEOREM 2. A subspace $E$ of $C(S)$ is normal if and only if it is weakly normal and $b w\left(E, M(S) / E^{0}\right)=\beta$.

In the proof we rely heavily on the following result of Rubel and Ryff [9, §2].

Proposition 4. On $C(S)$ the strict topology is stronger than the topology of uniform convergence on norm-null sequences in $M(S)$.

Proof. We repeat the argument of [9]. Let $A=\left(m_{n}\right)$ be a sequence in $M(S)$ which tends to zero in norm. We must show that, for some $k$ in $C_{0}(S)$ and $\varepsilon>0$,

$$
\left\{f:\|f\|_{k} \leqq \varepsilon\right\} \subset\left\{f:\left|\int f d m\right| \leqq 1 \text { for all } m \text { in } A\right\} .
$$

It is enough to show that there is a nonnegative $k$ in $C_{0}(S)$ with

$$
C=\sup \left\{\int k^{-1} d|m|: m \text { in } A\right\}<\infty .
$$

For then whenever $f \in C(S)$ and $\|f\|_{k} \leqq 1 / C$, we will have

$$
\left|\int f d m\right|=\left|\int(f k) k^{-1} d m\right| \leqq 1,
$$

which will complete the proof. 
It remains to prove (2.1). Each member of $A$ has sigma-compact support [1, Lemma 5], and at most a finite number have total variation $\geqq 1 / 2$. It follows that there is a compact subset $K_{1}$ of $S$ such that $|m|\left(S-K_{1}\right) \leqq \frac{1}{2}$ for each $m$ in $A$. Similarly, there is a compact set $K_{2}$ whose interior contains $K_{1}$ such that $|m|\left(S-K_{2}\right) \leqq \frac{1}{4}$ for each $m$ in $A$. Continuing in this fashion we obtain a sequence $\left(K_{n}\right)$ of compact sets such that $K_{n}$ is contained in the interior of $K_{n+1}$, and

$$
\sup \left\{|m|\left(S-K_{n}\right): m \text { in } A\right\} \leqq 2^{-n} \quad(n=1,2, \ldots) .
$$

Let $\left(p_{n}\right)$ be a sequence of positive numbers decreasing to 0 such that $p_{n} \geqq\left\|m_{n}\right\|$ $(n=1,2, \ldots)$ and $\sum\left(p_{n} 2^{n}\right)^{-1}<2$. It follows from Urysohn's Lemma that there is a nonnegative function $k$ in $C_{0}(S)$ vanishing outside $\bigcup_{n} K_{n}$ such that $k=p_{n}$ on $\partial K_{n}$, and $p_{n} \geqq k \geqq p_{n+1}$ on $K_{n+1}-K_{n}(n=1,2, \ldots)$. Thus for each fixed $n$,

$$
\begin{aligned}
\int k^{-1} d\left|m_{n}\right| & =\int_{K_{n}} k^{-1} d\left|m_{n}\right|+\sum_{j=n+1}^{\infty} \int_{K_{j+1}-K_{j}} k^{-1} d\left|m_{n}\right| \\
& \leqq\left\|m_{n}\right\| / p_{n}+\sum_{j=n+1}^{\infty}\left(p_{j} 2^{j}\right)^{-1} \\
& \leqq 1+\sum_{1}^{\infty}\left(p_{j} 2^{j}\right)^{-1}<3,
\end{aligned}
$$

which completes the proof.

Proof of Theorem 2. If $E$ is weakly normal and $b w\left(E, M(S) / E^{0}\right)=\beta$, then $\beta$ coincides with $\alpha=w\left(E, M(S) / E^{0}\right)$ on the unit ball $E_{1}$ of $E$. Thus $E_{1}$ is $\beta$-compact, so $E$ is normal. Conversely, if $E$ is normal then it is weakly normal; and it follows from Theorem A that $\alpha=\beta$ on every positive multiple of $E_{1}$ (recall that $\alpha \subset \beta$ on $C(S)$ ). Thus $\beta$ coincides with $\alpha$ on every bounded subset of $E$, so $\beta \subset$ $b w\left(E, M(S) / E^{0}\right)$. However it follows immediately from Proposition 4 and Theorem C that $b w\left(E, M(S) / E^{0}\right) \subset \beta$ for any weakly normal subspace $E$; which completes the proof.

We apply Theorem 2 to the examples discussed in the first section.

Corollary $1[9, \S 2]$. Let $E=H^{\infty}(G)$, and $M=M(G)$. Then in the pairing

$$
\langle f, m\rangle=\int f d m \quad(f \text { in } E, m \text { in } M),
$$

$E$ is isometric with the dual of $M / E^{0}$ and $\beta=b w\left(E, M / E^{0}\right)$. The same is true if $E=E(\Lambda)$ and $M=M\left(R^{n}\right)$.

We remark that this result, and its proof, remain valid when $G$ is a region in $C^{n}$.

Corollary 2. Let $\Gamma$ be an index set. Then $\beta=b w\left(l^{\infty}(\Gamma), l^{1}(\Gamma)\right)$.

Corollary 3. Let $E=\operatorname{Lip}(X, d)$ and $M=M(S)$. Then in the pairing

$$
\langle f, m\rangle=\int_{W} f d m \quad(f \text { in } E, m \text { in } M),
$$


$E$ is isometric to the dual of $M / E^{0}$, and $b w\left(E, M / E^{0}\right)$ coincides with the topology induced by the seminorms

$$
\|f\|_{k}=\max \left\{\|f\|_{\infty},\|\omega f\|_{k}\right\},
$$

where $k$ runs through $C_{0}(Y)$, and

$$
\|\omega f\|_{k}=\sup \{|\omega f(p) k(p)|: p \text { in } Y\} .
$$

We remark that the fact that $\operatorname{Lip}(X, d)$ is a conjugate Banach space is a special case of a result of J. A. Johnson [7, Theorem 4.1].

For a locally convex space $X$, the equicontinuous weak star topology ew $\left(X^{\prime}, X\right)$ is defined as the strongest topology on $X^{\prime}$ which coincides with $w\left(X^{\prime}, X\right)$ on equicontinuous sets $[6,5.3$, p. 61$]$. If $X$ is a Banach space then $e w\left(X^{\prime}, X\right)$ coincides with the bounded weak star topology. If $e w\left(X^{\prime}, X\right)$ coincides with the topology of uniform convergence on precompact subsets of $E$, then $X$ is called an $S$ space $[6,6.1$, p. 71]. It follows from [6, 6.6, Proposition 5] that in the bounded weak star topology, the dual of a Banach space is a complete $S$ space. Thus Corollary 2 provides another proof of Collins' observation $[2,4.6]$ that $\left(l^{\infty}(\Gamma), \beta\right)$ is a complete $S$ space. In fact, if $S$ is a locally compact Hausdorff space and $E$ is a normal subspace of $C(S)$, then it follows from these remarks and Theorem 2 that $(E, \beta)$ is a complete $S$ space.

Finally, we observe that in some sense the bounded weak star topology on the dual of a Banach space is always a strict topology.

Proposition 5. If $E$ is a Banach space, then there is a set $\Gamma$ and a norm isomorphism of $E^{\prime}$ onto a $w\left(l^{\infty}(\Gamma), l^{1}(\Gamma)\right)$-closed subspace $F$ of $l^{\infty}(\Gamma)$, which is simultaneously a topological isomorphism of $\left(E^{\prime}, b w\left(E^{\prime}, E\right)\right)$ onto $(F, \beta)$.

Proof. It is well known that there is a norm isomorphism taking $E$ onto a quotient space $l^{1}(\Gamma) / N$ for some $\Gamma$ (see [8, p. 283] for $E$ separable; the proof for nonseparable $E$ is similar). It follows upon passing to the adjoint that $E^{\prime}$ is norm isomorphic with $\left(l^{1}(\Gamma) / N\right)^{\prime}=N^{0}$, the annihilator of $N$ in $l^{\infty}(\Gamma)$. Thus $F=N^{0}$ is a $w\left(l^{\infty}(\Gamma), l^{1}(\Gamma)\right)$ closed subspace of $l^{\infty}(\Gamma)$, and it follows from Theorem $\mathrm{C}$ that the isomorphism between $E^{\prime}$ and $F$ stays bicontinuous when the spaces are given their respective bounded weak star topologies. But from Corollary $2, \beta=b w\left(l^{\infty}(\Gamma), l^{1}(\Gamma)\right)$, which coincides on $F$ with $b w\left(F, l^{1}(\Gamma) / N\right)$; and the proof is complete.

3. Hessler's Theorem. The following result is due to P. Hessler (unpublished).

THEOREM D. A subset of $H^{\infty}(G)$ is strictly closed if and only if it is strictly sequentially closed.

Rubel and Ryff have shown that Hessler's Theorem is a consequence of

THEOREM E [9, §2]. If $X$ is a separable Banach space, then a subset of $X^{\prime}$ is closed in the bounded weak star topology if and only if it is sequentially closed. 
Since the restriction of $w\left(X^{\prime}, X\right)$ to the $X^{\prime}$ unit ball is metrizable whenever $X$ is separable, Theorem $\mathrm{E}$ follows from the fact that a set is $b w\left(X^{\prime}, X\right)$-closed if and only if its intersection with every positive multiple of the unit ball is $w\left(X^{\prime}, X\right)$ closed. Theorem D follows from Theorem E, Corollary 1 of Theorem 2, and the fact that $M(G) / H^{\infty}(G)^{0}$ is separable $[10, \S 4]$.

In this section we use Theorem 2 to prove the following extension of Hessler's result.

THEOREM 3. If $S$ is separable and $E$ is a normal subspace of $C(S)$, then a subset of $(E, \beta)$ is closed if and only if it is sequentially closed.

This will follow from the next proposition, which is itself useful in applications.

Proposition 7. Let $E$ be a weakly normal subspace of $C(S)$, let $V$ be a norm closed subspace of $M(S)$, and let $E^{V}=E^{0} \cap V$. The following are equivalent:

(a) The mapping

$$
m+E^{v} \rightarrow m+E^{0} \quad(m \text { in } V)
$$

is a linear isometry of $V / E^{V}$ onto $M(S) / E^{0}$.

(b) $E$ is isometric to the dual of $V / E^{V}$ in the pairing

$$
\left\langle m+E^{v}, f\right\rangle=\int f d m \quad(f \text { in } E, m \text { in } V) .
$$

(c) For each $f$ in $E$,

$$
\|f\|_{\infty}=\sup \left\{\left|\int f d m\right|: m \text { in } V,\|m\| \leqq 1\right\} .
$$

Proof. Let $M=M(S)$, and let $E_{1}$ denote the unit ball of $E$. (a) $\rightarrow$ (b) follows immediately from Theorem 1 , and (b) $\rightarrow$ (c) is obvious.

(b) $\rightarrow$ (a). It follows from (b) and Theorem 1 that $\left\|m+E^{v}\right\|$ coincides with $\left\|m+E^{0}\right\|$ for each $m$ in $M$, so the mapping (3.1) is an isometry taking $V / E^{v}$ onto a closed subspace of $M / E^{0}$. But the image of $V / E^{V}$ is dense, since no nonzero linear functional on $M / E^{0}$ can annihilate it (Theorem 1 and (b) again). Thus (3.1) maps $V / E^{v}$ onto $M / E^{0}$.

(c) $\rightarrow$ (b). It follows from (c) that the mapping which takes each $f$ in $E$ to the linear functional

$$
m \rightarrow \int f d m \quad(m \text { in } V)
$$

is an isometry of $E$ into $V^{\prime}$. Since $w(E, V)$ is Hausdorff and weaker than $w(E, M)$ $=\alpha$, it follows from Theorem $\mathrm{A}$ and weak normality that $E_{1}$ is $w(E, V)$-compact, hence $w\left(V^{\prime}, V\right)$-compact. By Theorem B, $E$ is $w\left(V^{\prime}, V\right)$-closed, and as in the proof of Theorem 1, it is $w\left(V^{\prime}, V\right)$-dense. Q.E.D.

Proof of Theorem 3. Let $T$ be a countable dense subset of $S$, and let $V$ denote the space of measures in $M(S)$ which are concentrated on $T$. Clearly $V$ is a separable 
closed subspace of $M(S)$ satisfying (c) of Proposition 7. Thus $M(S) / E^{0}$ is isometrically isomorphic with $V / E^{v}$. Since $V / E^{v}$ is separable, so is $M(S) / E^{0}$, and the proof is completed by appealing to Theorem 2 and Theorem E.

We remark that the spaces $H^{\infty}(G), E(\Lambda), l^{\infty}$, and $\operatorname{Lip}(X, d)$ all satisfy the hypotheses of Theorem 3 . For $\operatorname{Lip}(X, d)$ we must show that the associated locally compact space $W=X \cup Y$ is separable. Since $X$ is a compact metric space, it is separable, as is $X \times X . Y$ is an open subset of $X \times X$, hence is separable; and $W$ is separable because it is the disjoint union of $X$ and $Y$.

A form of Proposition 7 was proved for $H^{\infty}(G)$ by Rubel and Shields [10, §4], who showed that if $E=H^{\infty}(G)$ and $V=L^{1}(G)$ then $E$ is isometric in the integration pairing to the dual of $V / E^{v}$. Note that in this case $V$ clearly satisfies (c) of Proposition 7. Similarly, taking $E=E(\Lambda)$ and $V=L^{1}\left(R^{n}\right)$, we obtain the same result.

\section{REFERENCES}

1. R. G. Buck, Bounded continuous functions on a locally compact space, Michigan Math. J. 5 (1958), 95-104. MR 21 \#4350.

2. H. S. Collins, On the space $l^{\infty}(S)$, with the strict topology, Math. Z. 106 (1968), 361-373. MR 39 \#763.

3. K. de Leeuw, Banach spaces of Lipschitz functions, Studia Math. 21 (1961/62), 55-66. MR 25 \#4341.

4. W. F. Donoghue, Jr., Distributions and Fourier transforms, Academic Press, New York, 1969.

5. N. Dunford and J. T. Schwartz, Linear operators. I: General theory, Pure and Appl. Math., vol. 7, Interscience, New York, 1958. MR 22 \#8302.

6. T. Husain, The open mapping and closed graph theorems in topological vector spaces, Clarendon Press, Oxford, 1965. MR 31 \#2589.

7. J. A. Johnson, Banach spaces of Lipschitz functions and vector-valued Lipschitz functions, Trans. Amer. Math. Soc. 148 (1970), 147-169.

8. G. Köthe, Topologische lineare Räume. I. Die Grundlehren der math. Wissenschaften, Band 107, Springer-Verlag, Berlin, 1966. MR 33 \#3069.

9. L. A. Rubel and J. V. Ryff, The bounded weak-star topology and the bounded analytic functions, J. Functional Analysis 5 (1970), 167-183.

10. L. A. Rubel and A. L. Shields, The space of bounded analytic functions on a region, Ann. Inst. Fourier (Grenoble) 16 (1966), fasc. 1, 235-277. MR 33 \#6440.

QUEEN'S UNIVERSITY, Kingston, Ontario, Canada

Michigan State University,

East Lansing, Michigan 48823 